\title{
Active surveillance for safety monitoring of seasonal influenza vaccines in Italy, 2015/2016 season
}

\author{
Stefania Spila Alegiani ${ }^{1 \dagger}$, Valeria Alfonsi ${ }^{2^{*}+} \mathbb{D}$, Eva Charlotte Appelgren ${ }^{2}$, Lorenza Ferrara ${ }^{3}$, Tolinda Gallo ${ }^{4}$, \\ Cristiano Alicino ${ }^{5}$, Maria Grazia Pascucci ${ }^{6}$, Silvia Aquilani ${ }^{7}$, Antonietta Spadea ${ }^{8}$, Silvio Tafuri ${ }^{9}$ and Caterina Rizzo ${ }^{10}$
}

\begin{abstract}
Background: Surveillance for adverse events following immunization is an important component of any national immunization programme because it is critical to assessing the safety of vaccines and to detecting potentially rare and severe adverse events and responding in a timely manner. We conducted an enhanced active surveillance aimed at assessing the safety of flu vaccines in the 2015-2016 season in Italy. The study was targeted to the population groups for which the seasonal vaccine is recommended in Italy.

Methods: During the study period, a total of 3213 individuals receiving seasonal influenza vaccination were registered on the web-based platform. Any adverse events experienced after 7 days from vaccination by individuals aged six months or more were collected through a telephone interview or by a web-based self-administered questionnaire. All individuals experiencing at least one event during the 7 days of follow-up were contacted for follow-up to 60 days.

Results: Overall, 854 events were reported: 845 events (26\%) after administration of the first dose and 9 (12\%) after the second dose. The majority of adverse events reported after 7 days from the first dose were of little clinical importance, and most involved local symptoms.

Conclusion: Our data, even though the number of vaccinated individuals was smaller than expected, is consistent with the safety of influenza vaccines in Italy during the 2015-2016 season regarding the most common adverse events. Further efforts are needed to obtain sufficient power to study rarer adverse events. Active monitoring and systematic studies to test generated signals and hypotheses are crucial to intensify awareness among the public and professionals with regard to the safety of vaccines.
\end{abstract}

Keywords: Influenza, Vaccines, Safety, Surveillance

\section{Background}

Influenza is a major public health problem; a conservative estimate suggests that the illness infects every third child and every tenth adult, annually 60 million of the 500 million inhabitants of the European Union. It is related to 50 million episodes of mild clinical disease, 150 thousand hospital admissions and 15 to 40 thousand deaths annually $[1-5]$.

\footnotetext{
* Correspondence: valeria.alfonsi@iss.it

†Stefania Spila Alegiani and Valeria Alfonsi contributed equally to this work. ${ }^{2}$ Department of Infectious Disease, Istituto Superiore di Sanità - Italian National Institute of Health, Viale Regina Elena 299, 00161 Rome, Italy Full list of author information is available at the end of the article
}

Vaccines are the cornerstone of prevention for influenza and its consequences [6]. However, in the last two decades, controversies regarding the effectiveness and safety of influenza vaccines have triggered extensive epidemiological research and networking, an effort that is further strengthened by the need for pandemic preparedness [7].

In Italy, seasonal influenza vaccination is recommended and provided free of charge to the elderly ( $\geq 65$ years) and to people aged 6 months to 64 years with comorbidities that increase the risk of influenza complications and to those in other categories (e.g., pregnant women in the second and third trimester, health care workers, etc.) [8]. 
Vaccine safety surveillance is passively undertaken by the spontaneous reporting system of the Italian national pharmacovigilance system, through the collection and analysis of adverse events following immunization (AEFI) based on spontaneous reports from doctors, health professionals and patients. Through these reports may emerge signs that suggest a need for supplementary investigation to deny or confirm issues and to quantify them in terms of risk through ad hoc pharmacoepidemiology studies.

Therefore, pharmacovigilance is mostly inadequate in its ability to early detect events; a renewed approach is essential and necessary to quickly and effectively track vaccinerelated AEFI and to provide reliable, near real-time data and denominators on numbers of those vaccinated. Additionally, such types of studies also allow an assessment of the safety profile of the vaccine by product and brand, unlike pharmacovigilance, which is lacking in denominators and suffers from under reporting. Accordingly, the European Medicines Agency (EMA) has released regulatory guidance on enhanced safety surveillance for seasonal influenza vaccines in the EU [9].

In this regard, pharmacoepidemiology studies conducted in different populations to evaluate influenza vaccine safety show that local side effects, such as swelling, redness and pain at the injection site, which generally are mild and rarely interfere with daily activities, are common, occurring in more than $10 \%$ of recipients. Fever, tiredness and myalgia also occur commonly (1$10 \%)$. These adverse events may be more pronounced in children [10-13].

The Italian National Institute of Health (ISS) has the experience and organization necessary to conduct influenza vaccine effectiveness and safety studies at the national level through the involvement of local health units (LHU), general practitioners (GPs) and paediatricians, using ad hoc tools $[10,14-16]$.

This paper presents findings from a study conducted during the 2015-2016 season in Italy, targeted to the population group for which the influenza vaccine is recommended and aimed at implementing enhanced surveillance to evaluate influenza vaccine safety.

\section{Methods}

\section{Study setting and population}

An invitation letter was sent through Italian interregional prevention coordination to vaccine centres (GPs, paediatricians, hospitals and vaccine services of LHU) in 6 selected Italian Regions (Piedmont, Liguria, Friuli Venezia-Giulia, Emilia Romagna, Lazio, Apulia) for involvement in the study.

All individuals aged 6 months or more receiving the seasonal influenza vaccine in vaccine centres who spontaneously approached the vaccine centres were asked to participate. Eligible participants were those who provided signed written informed consent (parental consent was obtained for individuals aged $<18$ years) and who agreed to be interviewed by telephone or to fill in a web-based questionnaire 7 days after vaccination.

\section{Organization of the study}

Information on vaccinated individuals as well as regarding vaccine brand, date of vaccination, medical conditions, and self-reported history of allergy and influenza vaccination in the previous season were collected, during the medical pre-vaccination assessment, through a case report form (CRF, Additional file 1) by vaccine centres. Then, each recruited individual was given a diary to record any event or reaction and healthcare consultation that occurred within 7 days of vaccination, including visit to a GP, access to an emergency department or hospitalization.

After 7 days (follow-up to 7 days according to the EMA guidelines [9]), all parents (in the case of subjects aged less than 18 years old) and vaccinated individuals were asked to communicate any AEFIs reported in their diary during a telephone interview. In one region (Emilia Romagna) it was also proposed that participants directly enter these data on a web-based platform. All information was collected through a standardized questionnaire that included a list of events in a drop-down menu ( $\mathrm{Y} /$ $\mathrm{N})$ and a free text option for other responses not included in the menu.

AEFIs included local events, such as redness, pain, and indurations, and systemic events, such as fever (above $37.5^{\circ} \mathrm{C}$ ), chills, myalgia, arthralgia, general weakness, headache, syncope, nausea, and vomiting. Serious adverse events (SAE) were categorized according to predefined criteria, which included any unexpected medical event that resulted in death, was life-threatening or required hospitalization [17]. We also included seizures requiring medical attendance (emergency department vi sits and/or hospitalization) as medically important events.

All individuals experiencing at least one event during the 7 days of follow-up were contacted by telephone 60 days after vaccination to monitor the course of the e vents (follow-up to 60 days).

Data for the study were compiled from mid October 2015 through March 2016.

Data were entered into a web-based database, which was protected in terms of security. The enrolment and input of information from the vaccinated individuals was monitored through the web-based platform to highlight in real time possible errors or data quality issues (i.e., regarding date of vaccination, name of vaccine, outcome of the follow up).

Suspected adverse reactions observed during follow-up of the vaccinated individuals were reported to the $\mathrm{Na}$ tional Network of Pharmacovigilance by vaccine centres 
using a standard form printed directly from the webbased platform.

The study was approved by the Ethical Committee of the National Institute of Health.

\section{Statistical analysis}

The frequency of reported AEFIs was computed as the ratio between vaccinated individuals who reported at least one event and the total number of vaccinated in dividuals.

The association between the characteristics of participants and the type of vaccine and the occurrence of at least one event during the 7 days of follow-up was analysed through a Chi-square test for categorical variables. The multivariate logistic regression model was performed taking into account all potential confounding factors available from the CRF. In the final model, we included all variables with $p<0.05$ in the univariate analysis, excluding influenza vaccination in the campaign 2014-2015 because of the large amount of missing of data. SPSS software (IBM SPSS Statistics, version 22) was used for the statistical analyses.

\section{Results}

A total of 9 GPs, 8 paediatricians, 2 hospitals and 13 vaccine services of LHUs, distributed in 6 Italian Regions participated in the study (Fig. 1).

Seven different available commercial products were included in the study: two subunit vaccines, three split vaccines, one adjuvanted vaccine and one intradermal vaccine. Only subunit vaccines were used by all participating regions; split vaccines were used in 4 regions and adjuvanted and intradermal vaccines in 3 regions.
During the study period, a total of 3213 individuals receiving seasonal influenza vaccination, who provided written informed consent, were registered into the web-based platform, 73 of whom received two doses of vaccine (Fig. 2).

The median age of the study population was 57 years (range 8 months to 100 years), $20 \%$ were paediatric patients aged 6 months- 14 years, $4 \%$ were aged $15-25$ years and $38 \%$ were more than 65 years old; the male-to-female ratio was 1 (1614 males and 1599 females). Half of the participants received a subunit vaccine $(n=1636)$, $21 \%(n=660)$ a split vaccine, $16 \%(n=526)$ an adjuvanted vaccine and $12 \%(n=394)$ an intradermal vaccine.

The majority of subjects were high-risk individuals (70\%); this included those who were older, pregnant women and those with chronic health conditions who are the target of the influenza vaccination programme in Italy [8]. Approximately $40 \%$ of these individuals had an underlying medical condition, mainly cardiovascular and respiratory in nature. A total of 1958 participants (61\%) had also been vaccinated against influenza in the previous season (2014-2015).

The number of telephone interviews performed for the 7-day follow-up was 3152, and 49 vaccinated individuals directly entered data on the web-based platform; therefore, the follow-up to assess any event within 7 days of vaccination was completed for $97 \%(n=3201)$ of vaccinated individuals. All the 49 vaccinated participants entering data via web were recruited from the Emilia Romagna region, the only one that decided to apply the web-based approach, and they were younger (median age 53 vs 58 in the study population) and mainly

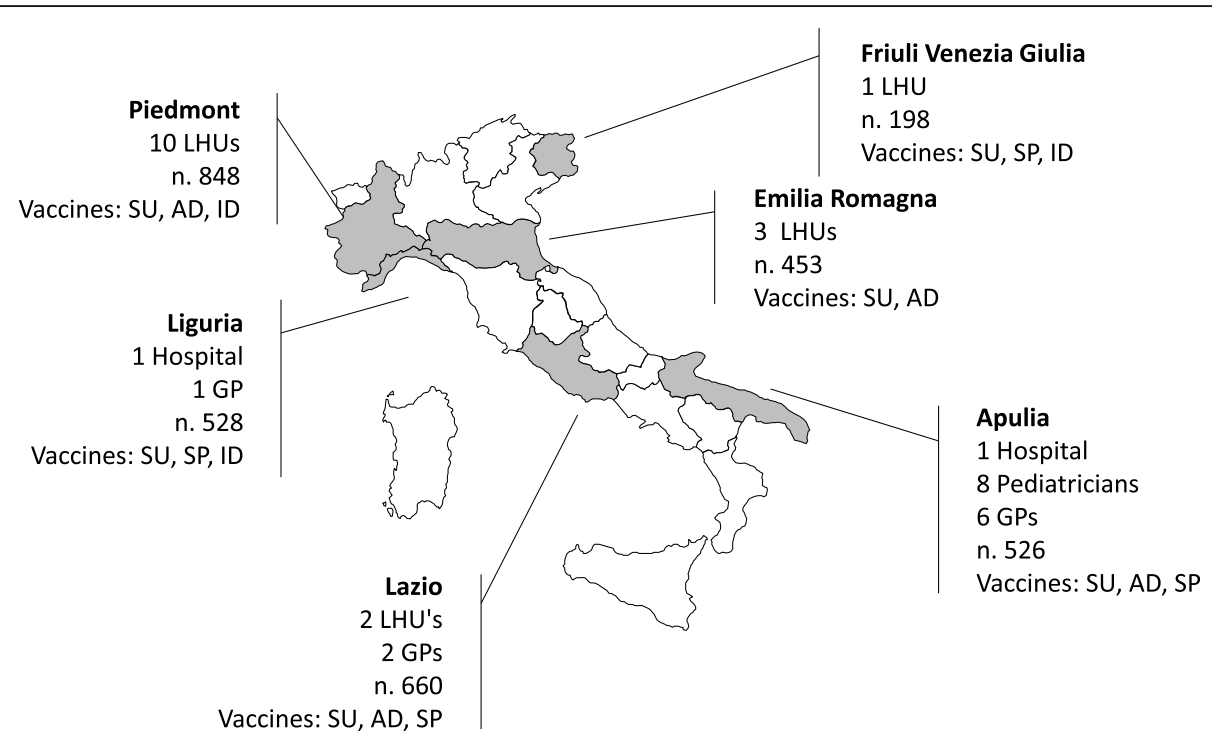

Fig. 1 Number of centres, number of vaccinated subjects and type of vaccines by region. LHU: local health unit; GP: general practitioner; SU: subunit; AD: adjuvanted; SP: split; ID: intradermal 


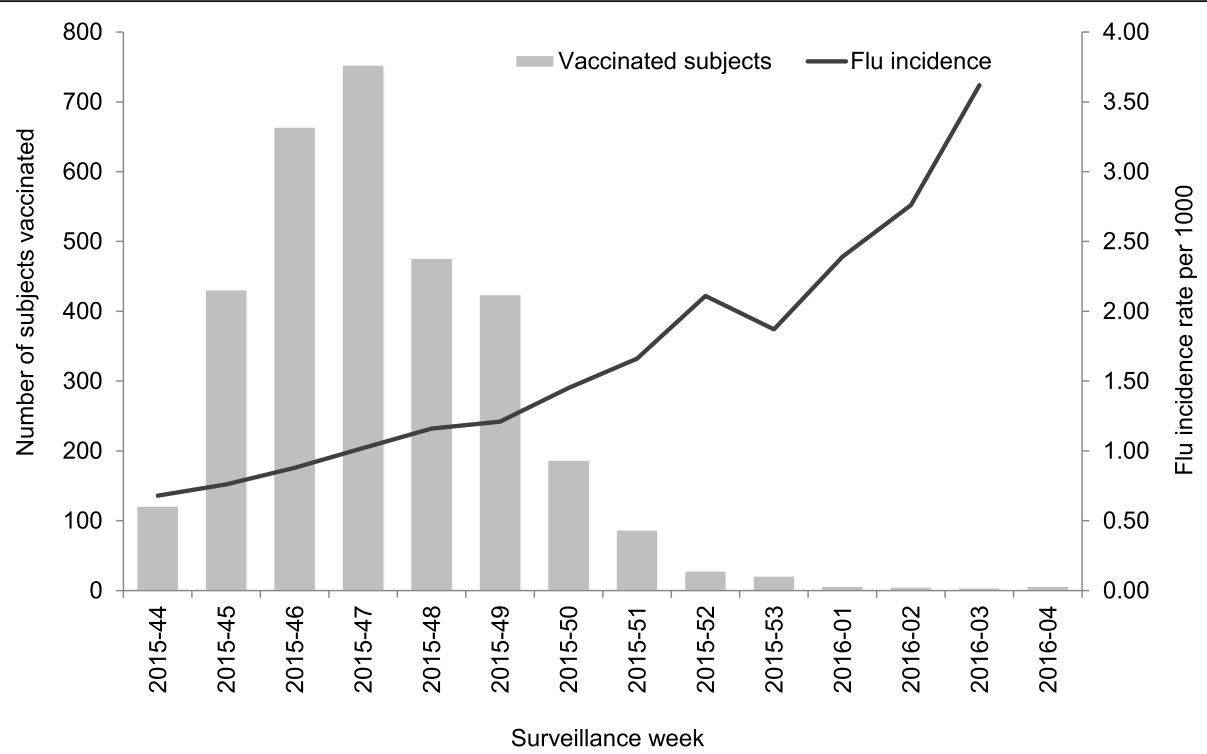

Fig. 2 Number of vaccinated subjects and flu incidence by week of vaccination, 2015-2016 season

vaccinated with the subunit vaccine $(86 \%)$ as was the vaccine available for this age group.

Overall, 854 AEFIs were reported: 845 events (26\%) after administration of one dose and $9(12 \%)$ after the two doses. The proportion of AEFIs reported varied between 21 and $22 \%$ of the split and adjuvanted vaccines to $27 \%$ of the subunit vaccines and $38 \%$ of the intradermal vaccine (Table 1). The majority of AEFIs reported 7 days from vaccination were of little clinical importance (Table 2), except for two SAE, which occurred after the administration of split vaccines. These involved two elderly individuals (a male age 76 and a female age 87) with severe chronic underlying conditions who died three days (during a hospitalization) and ten days after the vaccination, respectively. No further hospitalizations were reported but one ED visit for conjunctivitis was noted. The most frequent AEFIs reported were local symptoms (pain, $n=446$; redness, $n=222$; swelling, $n=$ 209; and induration, $n=174)$, followed by malaise $(n=$ $122)$, fever $\geq 37.5^{\circ} \mathrm{C}(n=75)$, headache $(n=72)$ and arthralgia $(n=42)$, with no significant differences for type of vaccines administered (Table 2 ).

Younger ages (OR 1.98; 95\% CI 1.46-2.68), females (OR 1.39; 95\% CI 1.19-1.63) and individuals not vaccinated against influenza in the previous season (OR 1.28; $95 \%$ CI 1.07-1.54) reported significantly higher rates of events within 7 days of vaccination (Table 1 ).

In the multivariate logistic regression model age, sex and type of vaccine were associated with the occurrence of any event within 7 day of vaccination (Table 3). Assuming the subunit vaccines as reference category, the adjusted ORs were higher for intradermal vaccines (OR 3.53; 95\% CI 2.58-4.84) and adjuvanted vaccines (OR
1.81; 95\% CI 1.31-2.49). The multivariate analysis confirmed the highest rate of AEFIs occurred in younger vaccinated individuals (age $\leq 14$ and $15-25$ years) compared to those older than 75 years (OR 3.80; 95\% CI 2.55-5.67 and OR 3.26; 95\% IC 1.77-6.00, respectively) and in females compared to males (OR 1.51; 95\% CI 1.27-1.80) (Table 3).

Among the 854 vaccinated subjects experiencing at least one AEFI within 7 days of vaccination, 829 subjects (97\%) were contacted during the follow-up period at 60 days; 4 subjects reported that events were not fully resolved, while 25 subjects (2.9\%) reported new AEFIs, most of them respiratory conditions.

\section{Discussion}

Surveillance of adverse events following immunization is an important component of any national immunization programme because it is critical to assessing the safety of vaccines and to detecting potentially rare and severe adverse events and responding in a timely manner. This is even truer regarding the influenza vaccine because it is reformulated each year and annual revaccination is recommended. As available seasonal influenza vaccines safety profiles may vary between types and products and from year to year, active surveillance is crucial to monitor the possibility of adverse events occurring following manufacturing changes, contamination of batches or through the introduction of new pandemic influenza strains.

As seasonal influenza vaccines present several specific challenges for pharmacovigilance, more emphasis should be placed on the post-licensure monitoring of the benefit/risk of influenza vaccines, including a request for 
Table 1 Vaccinated individuals with at least one event within 7 days of vaccination by geographical area, type of vaccine and characteristics

\begin{tabular}{|c|c|c|c|}
\hline & \multicolumn{2}{|c|}{ Number of subjects vaccinated ${ }^{a}$} & \multirow[b]{2}{*}{ OR $(95 \% \mathrm{Cl})$} \\
\hline & Total & With any event (\%) & \\
\hline \multicolumn{4}{|l|}{ Geographical area } \\
\hline Nord-west (Piedmont, Liguria) & 1376 & $352(25.6)$ & 1 \\
\hline Nord-east (Friuli Venezia Giulia, Emilia Romagna) & 651 & $203(31.2)$ & $1.32(1.07-1.62)$ \\
\hline Centre-South (Lazio, Apulia) & 1186 & $290(24.5)$ & $0.94(0.79-1.13)$ \\
\hline \multicolumn{4}{|l|}{ Type of vaccines ${ }^{b}$} \\
\hline Subunit (2) & 1636 & $444(27.1)$ & 1 \\
\hline Adjuvanted (1) & 526 & $114(21.7)$ & $0.74(0.59-0.94)$ \\
\hline Split (3) & 660 & $138(20.9)$ & $0.71(0.57-0.88)$ \\
\hline Intradermal (1) & 394 & $149(38.1)$ & $1.65(1.31-2.08)$ \\
\hline \multicolumn{4}{|l|}{$\mathrm{Age}^{\mathrm{c}}$} \\
\hline$\leq 14$ & 651 & $208(32.0)$ & $1.98(1.46-2.68)$ \\
\hline $15-25$ & 142 & $70(49.3)$ & $4.10(2.71-6.22)$ \\
\hline $26-65$ & 1174 & $310(26.4)$ & $1.51(1.14-2.02)$ \\
\hline $66-79$ & 854 & $183(21.4)$ & $1.15(0.85-1.56)$ \\
\hline$\geq 80$ & 381 & $73(19.2)$ & 1 \\
\hline \multicolumn{4}{|l|}{ Sex } \\
\hline Males & 1614 & $373(23.1)$ & 1 \\
\hline Females & 1599 & $472(29.5)$ & $1.39(1.19-1.63)$ \\
\hline \multicolumn{4}{|l|}{ Seasonal vaccination, $2014-2015^{d}$} \\
\hline Yes & 1958 & $490(25.0)$ & 1 \\
\hline No & 805 & $241(29.9)$ & $1.28(1.07-1.54)$ \\
\hline \multicolumn{4}{|l|}{ High risk condition ${ }^{e}$} \\
\hline No & 454 & $134(29.5)$ & 1 \\
\hline Yes & 2253 & $581(25.8)$ & $0.83(0.66-1.04)$ \\
\hline Total & 3213 & $845(26.3)$ & \\
\hline
\end{tabular}

${ }^{a}$ vaccinated with one dose of flu vaccine

${ }^{\mathrm{b}}$ number of different products in brackets

${ }^{\mathrm{C}}$ Age not available for 11 subjects

${ }^{\mathrm{d}}$ Seasonal vaccination for 2014-2015 not available for 542 subjects

e Information on high risk condition not available for 506 subjects. High risk conditions include older people, pregnant women and people with chronic health

conditions; these are the target for the influenza vaccination programme in Italy. [8]

OR odds ratio, $\mathrm{Cl}$ confidence interval

continuous monitoring of efficacy and enhanced safety surveillance that needs to be tailored according to where it is used.

This study aimed at assessing the safety of flu vaccines in the 2015-2016 season, through a protocol for active enhanced surveillance of AEFI. All individuals who approached the vaccine centres were invited to participate in the study. More than 3200 subjects, identified by the participating regions, were enrolled. However, this study has an important limitation: the adherence of vaccinated individuals was less than expected; therefore, it did not reach sufficient power to assess rarer AEs. In fact, the total number of recruited subjects is a very small proportion of the more than 3 million of vaccine doses administered in the participating regions during this season. This suggests that more efforts are needed in order to obtain sufficient numbers to identify rare events and to make comparisons between different vaccine brands or specific age groups. In the majority of cases, potential participants did not respond to recruitment efforts because of a general lack of interest and, for some, because of difficulties in communicating the information required during the consent process and in overcoming general resistance to participation in research studies. To encourage patient interest, approaching them one-on-one in their vaccine centre to inform about them about the project and developing additional strategies for follow up is crucial. Moreover, a 
Table 2 Distribution of events within 7 days of vaccination by type of vaccine

\begin{tabular}{|c|c|c|c|c|c|}
\hline \multirow[t]{2}{*}{ Events } & \multicolumn{5}{|c|}{ Number of vaccinated subjects ${ }^{a, b}$} \\
\hline & Subunit & Split & Adjuvanted & Intradermal & Total \\
\hline \multicolumn{6}{|l|}{ Local Symptoms } \\
\hline Local pain & 242 & 97 & 67 & 40 & 446 \\
\hline Local redness & 81 & 22 & 13 & 106 & 222 \\
\hline Local swelling & 93 & 26 & 15 & 75 & 209 \\
\hline Local Induration & 84 & 19 & 14 & 57 & 174 \\
\hline \multicolumn{6}{|l|}{ Systemic Symptoms } \\
\hline Malaise & 64 & 15 & 23 & 20 & 122 \\
\hline Fever $\geq 37.5^{\circ} \mathrm{C}$ & 48 & 12 & 8 & 7 & 75 \\
\hline Headache & 53 & 6 & 7 & 6 & 72 \\
\hline Arthralgia & 20 & 5 & 10 & 7 & 42 \\
\hline Vomiting and nausea & 20 & 6 & 4 & 6 & 36 \\
\hline Myalgia & 14 & 15 & 6 & 3 & 28 \\
\hline Loss of appetite & 15 & 6 & 1 & 3 & 25 \\
\hline Generalized itching & 11 & 1 & 1 & 8 & 21 \\
\hline Irritability & 13 & 0 & 0 & 0 & 13 \\
\hline Dyspnoea & 5 & 2 & 0 & 3 & 10 \\
\hline Conjunctivitis & 2 & 1 & 3 & 1 & 7 \\
\hline Rash & 3 & 0 & 0 & 2 & 5 \\
\hline Persistent, inconsolable crying lasting $\geq 3 \mathrm{~h}$ & 1 & 0 & 0 & 0 & 1 \\
\hline Urticaria & 0 & 0 & 0 & 0 & 1 \\
\hline Others & 44 & 4 & 10 & 25 & 83 \\
\hline
\end{tabular}

${ }^{a}$ Vaccinated with one dose of flu vaccine

${ }^{b}$ Total events may not equal the sum of individual symptoms reported, as vaccinated subjects were allowed to report multiple symptoms

Table 3 Logistic regression analysis of variables associated with any event within 7 days of vaccination among 3213 subjects

\begin{tabular}{ll}
\hline & Adjusted OR (95\% Cl) \\
\hline Age & \\
$15-25$ & $3.80(2.55-5.67)$ \\
$26-65$ & $3.26(1.77-6.00)$ \\
$66-79$ & $2.45(0.90-1.72)$ \\
$\geq 80$ & $1.51(1.17-1.80)$ \\
Sex & 1 \\
Males & \\
Females & 1 \\
Type of vaccines & $1.51(1.27-1.80)$ \\
Subunit & \\
Adjuvanted & 1 \\
Split & $1.81(1.31-2.49)$ \\
Intradermal & $0.71(0.53-0.96)$ \\
\hline
\end{tabular}

Variables considered in the multivariate model are age at vaccination, sex and type of vaccines administered

OR odds ratio, $\mathrm{Cl}$ confidence interval variety of strategies to make potential participants aware of the study, including sending letters from the patient's GPs and distributing flyers on influenza vaccination, could be useful. Furthermore, recently in Italy, there is a widespread skepticism and great hesitancy about vaccination, most of which is due to uncertainties regarding the safety profile of influenza vaccines [18].

Some variability by region regarding the number of those vaccinated was observed: this variation may depend on several factors, such as the size of the resident population, the vaccine coverage and, mainly, on the number and type of the vaccine centres participating to the study. In Italy, vaccine coverage in the general population (not just in all the target groups) in the 20152016 season was equal to $13.9 \%$, and coverage in the participating regions ranged from 13.6 to $16.1 \%$.

Our study aimed to actively follow-up defined cohorts of children, adults and the elderly at 7 days after immunization for specific AEFIs. The observed frequency of AEFIs that occurred within 7 days of influenza vaccination (26\%), corresponded to other similar surveys conducted on elderly or paediatric populations [10-13], and it was consistent with the safety data of the vaccines [19]. These events were all mild, except for two deaths 
after administration of the influenza vaccine in subjects with underlying chronic conditions; clinicians excluded the correlation with the vaccination, according to the WHO criteria for AEFI [20]. Our study assessed the slightly higher risk for adjuvant and intradermal vaccines compared to the subunit and split (similar risks) vaccines, in line with Product Data Sheets and other comparison studies [21].

Stratified analysis showed variability in the frequency of mild events by type of vaccine and characteristics of the vaccinated individuals. The follow-up at 60 days of the 854 vaccinated individuals who reported at least one AEFI revealed a low occurrence of new symptoms (3\%), and these were mainly respiratory and gastrointestinal symptoms. In 4 cases, however, the symptoms that occurred within the first 7 days continued to persist.

The study relied on two different methods to collect events recorded in the vaccine diary: the first method relied on a telephone interview with a vaccine centre operator and the second method directly involved the vaccinated person (or the parent, in the case of child) on a web platform. The web platform was tested at ISS and was shown to perform well, be easy to use and to provide good compliance; but, in this surveillance, fewer than $2 \%$ of the vaccinees used the web platform for data entry. This may be due to the lack of familiarity with informatics tools in those over the age 65 years, a group that made up $40 \%$ of the study population; the time was lacking to explain in more in detail the data entry system of health workers during the vaccine sessions. Although the additional information required during the vaccination was limited, we observed $14 \%$ of the data was missing, mainly concerning previous vaccinations, previous allergies, chronic illnesses and risk conditions. All this information is routinely gathered during the vaccination session but they are collected on paper and not always included in the IT (Information technology) sys tems available at vaccination centres. Therefore, automatic information collection systems should be adopted in this type of study to reduce operator workload and improve the quality of collected data. Otherwise, automated SMS-based reporting can facilitate sustainable, near real-time monitoring of adverse reactions and contribute to early identification of potential vaccine safety issues [22, 23]. Active surveillance of AEFI using SMS potentially permits more rapid identification of emerging safety signals. Also, compared with voice telephone interviews, data collection by SMS results in significantly improved response rates and timeliness of vaccine safety data [24].

However, our enhanced surveillance ensured to rapidly detect changes in the frequency and/or severity of expected reactogenicity that may indicate a potential for more serious risks as exposure to the vaccine increases.
Moreover, it was able to generate the results during the entire vaccination campaign (October-December), as those vaccinated were enrolled and follow-up data was gathered 7 days after immunization.

\section{Conclusions}

In conclusion, our data, even though they refer to a limited number of vaccinated subjects, are consistent with the safety of influenza vaccines in Italy during the 20152016 season for the most common adverse events. This could make a positive contribution towards improving the capability of public health systems to respond to demands concerning the safety surveillance system, even when the EMA guideline requirements were not closely met.

Mere registration and passive surveillance system reporting of possible adverse reactions are not enough to sustain confidence in the safety of the vaccines [25]. Active monitoring and systematic studies are important to test generated signals and hypotheses and to intensify awareness of the public and professionals with regard to the safety of vaccines [26].

To obtain enough power for comparative safety analyses, we are planning for next year's season large cohort studies to identify long-term and rare adverse events through record linkage of different health databases. Alternatively, other approaches requiring only cases, some of which may use self-controlled techniques, can be applied [27-29].

\section{Additional file}

Additional file 1: Case report forms of the study. Case report forms to collect information during vaccine administration, at 7 and 60 days after vaccine administration. (PDF $425 \mathrm{~kb}$ )

\section{Abbreviations}

AEFI: Adverse events following immunization; Cl: Confidence interval; CRF: Case report form; ED: Emerging department; EMA: European Medicines Agency; GP: General practitioners; ISS: Italian National Institute of Health; LHU: Local health units; OR: Odds ratio; SAE: Serious adverse events; SMS: Short message service

\section{Acknowledgements}

We wish to thank The Sveva Group* for their support and collaboration in regard to the conduct of the study at local level.

*The Sveva Study Group. Piedmont: Lorenza Ferrara; Friuli Venezia Giulia: Tolinda Gallo, Alessandro Cuccu, Giulia Degani, Daniela Gnesutta, Stefano Miceli, Giovanni Pilutti, Alice Pischiutti, Cristina Uliana; Liguria: Cristiano Alicino, Pierclaudio Brasesco, Valeria Faccio, Giancarlo Icardi, Sergio Schiaffino; Emilia Romagna: Maria Grazia Pascucci, Marisa Cova, Sara Ferioli, Licia Maniscalco, Monica Mascellani, Alma Nieddu, Roberto Rangoni; Lazio: Silvia Aquilani, Antonietta Spadea, Alessandro Bevilacqua, Patrizia Carpentieri, Anna Maria Casciani, Simonetta Casini, Emanuela Conversi, Anna Maria D’Amici, Marco Dari, Silvia Dari, Emanuele Di Cosimo, Lucia Donno, Eugenio Epifanio, Anna Rita Ferrero, Fabio Forleo, Gabriella Gozzi, Orazio Grimaldi, Fiammetta lometti, Domenica Ludovici, Anna Maria Matarrese, Patrizia Operamolla, Daniele Piras, Sergio Rogai, Fannina Scipione, Anna Valentini, Carmen Vinci; Apulia: Silvio Tafuri, Antonella Anaclerio, Viviana Balena, Monica Boffi, Maria Campolungo, Teresa Cazzato, Gaetano D’Ambrosio, Daniela Damiani, Mario 
Domenico Dell'Orco, Mario Lucio Dell'Orco, Bartolomeo Fanizza, Vincenzo Frappampina, Maria Filomena Gallone, Maria Serena Gallone, Cinzia Germinario, Francesco Mariani, Carmen Martino, Tecla Mastronuzzi, Ermanno Praitano, Michele Quarto, Angela Tricarico.

We would like to thank Giuseppe Pimpinella, Carmela Santuccio and Francesco Trotta (Italian Medicines Agency, AIFA) for their contribute to the initial discussion of the study design.

\section{Funding}

Only public employees of the national or regional health authorities were involved in conceiving, planning, and conducting the study; financial support was received from the Italian Medicines Agency (AIFA). The corresponding author had full access to all study data and approved the manuscript for publication

\section{Availability of data and materials}

The datasets used and analysed during the current study are available from the corresponding author on reasonable request. The datasets generated and analysed during the current study are not publicly available. The webbased database is password-protected and accessible only by study group members].

\section{Authors' contributions}

SSA acted as study supervisor, contributed to the study design, analysed the data and drafted the manuscript as the lead writer. VA drafted the manuscript and contributed equally with SSA to this article. CR contributed to the study design and commented on the manuscript. ECA made substantial contribution to acquisition of data at national level and was involved in revising the manuscript. LF, TG, CA, MGP, SA, AS, ST made substantial contribution to acquisition of data at regional level and advised on analyses and were involved in revising the manuscript. All the authors read and approved the final manuscript.

\section{Ethics approval and consent to participate}

The study was approved by the Ethical Committee of the Italian National Institute of Health (Prot PRE-C-317/15; 12/05/2015). All participants provided informed written consent prior to participate to the study.

\section{Consent for publication}

Not applicable

\section{Competing interests}

The authors declare that they have no competing interests.

\section{Publisher's Note}

Springer Nature remains neutral with regard to jurisdictional claims in published maps and institutional affiliations.

\section{Author details}

${ }^{1}$ National Center for Drug Research and Evaluation, Istituto Superiore di Sanità, Rome, Italy. ${ }^{2}$ Department of Infectious Disease, Istituto Superiore di Sanità - Italian National Institute of Health, Viale Regina Elena 299, 00161 Rome, Italy. ${ }^{3}$ SeREMI, Local Health Unit of Alessandria-Piedmont Region, Alessandria, Italy. ${ }^{4}$ Department of Prevention, Local Health Unit 4 Medio Friuli, Udine, Italy. ${ }^{5}$ Department of Health Sciences, University of Genoa, Genoa, Italy. ${ }^{6}$ Directorate General for Health and Social Policy Emilia-Romagna Region, Bologna, Italy. ${ }^{7}$ Viterbo Local Health Unit, Lazio Region, Viterbo, Italy. ${ }^{8} \mathrm{RM} / 1$ Local Health Unit, Lazio Region, Rome, Italy. ${ }^{9}$ Department of Biomedical Science and Human Oncology, University of Bari, Bari, Italy. ${ }^{10}$ Unit of Innovation and Clinical Pathways, Bambino Gesù Children's Hospital, Rome, Italy.

\section{Received: 7 February 2018 Accepted: 26 November 2018} Published online: 22 December 2018

\section{References}

1. Molbak K, Espenhain L, Nielsen J, Tersago K, Bossuyt N, Denissov G, et al. Excess mortality among the elderly in European countries, December 2014 to February 2015. Euro Surveill. 2015;20(11). https://doi.org/10.2807/15607917.ES2015.20.11.21065.
2. Jernigan DB, Cox NJ. Human influenza: One health, one world. In: Webster RG, Monto AS, Braciale TJ, Lamb RA, editors. Textbook of Influenza. 2nd ed. Oxford UK: John Wiley; 2013. p. 3-19.

3. Hayward AC, Fragaszy EB, Bermingham A, Wang L, Copas A, Edmunds WJ, et al. Comparative community burden and severity of seasonal and pandemic influenza: results of the flu watch cohort study. Lancet Respir Med 2014;2:445-5

4. Reed C, Meltzer MI, Finelli L, Fiore A. Public health impact of including two lineages of influenza $B$ in a quadrivalent seasonal influenza vaccine. Vaccine. 2012;30:1993-8.

5. Donaldson LJ, Rutter PD, Ellis BM, Greaves FE, Mytton OT, Pebody RG, et al. Mortality from pandemic a/H1N1 2009 influenza in England public health surveillance study. BMJ. 2009;339:5213.

6. World Health Organization. Vaccines against influenza WHO position paper - November 2012. Wkly Epidemiol Rec. 2012;87:461-76.

7. Kissling E, Nunes B, Robertson C, Valenciano M, Reuss A, Larrauri A, et al. I-MOVE multicentre case-control study 2010/11 to 2014/15: Is there withinseason waning of influenza type/subtype vaccine effectiveness with increasing time since vaccination? Euro Surveill. 2016;21(16). https://doi.org/ 10.2807/1560-7917.ES.2016.21.16.30201.

8. Ministero della Salute. Ministry of Health. Prevenzione e controllo dell'influenza: Raccomandazioni per la stagione 2015-2016 [Prevention and control of influenza: reccomendation for the 2015/2016 season] [in Italian]. Italy: Ministry of Health; 2015. http://www.trovanorme.salute.gov.it/norme/ renderNormsanPdf?anno=0\&codLeg $=52703 \&$ parte $=1 \% 20 \&$ serie $=$.

9. European Medicines Agency (EMA) Pharmacovigilance Risk Assessment Committee (PRAC). Interim guidance on enhanced safety surveillance for seasonal influenza vaccines in the EU. London: EMA; 2014. http://www.ema. europa.eu/docs/en_GB/document_library/Scientific_guideline/2014/04/ WC500165492.pdf

10. Spila-Alegiani S, Salmaso S, Rota MC, Tozzi AE, Raschetti R. Reactogenicity in the elderly of nine commercial influenza vaccines: results from the Italian SVEVA study. Study for the evaluation of adverse events of influenza vaccination. Vaccine. 1999;17(15-16):1898-904.

11. Pillsbury A, Cashman P, Leeb A, Regan A, Westphal D, Snelling T, et al. Realtime safety surveillance of seasonal influenza vaccines in children, Australia, 2015. Euro Surveill. 2015;20(43). https://doi.org/10.2807/1560-7917.ES.2015. 20.43.30050

12. Newes-Adeyi G, Greece J, Bozeman S, Walker DK, Lewis F, Gidudu J. Active surveillance for influenza vaccine adverse events: the integrated vaccine surveillance system. Vaccine. 2012;30(6):1050-5.

13. Cashman P, Moberley S, Dalton C, Stephenson J, Elvidge E, Butler M, et al. Vaxtracker: active on-line surveillance for adverse events following inactivated influenza vaccine in children. Vaccine. 2014;32(42):5503-8.

14. Rizzo C, Bella A, Alfonsi V, Puzelli S, Palmieri AP, Chironna M, et al. Influenza vaccine effectiveness in Italy: age, subtype-specific and vaccine type estimates 2014/15 season. Vaccine. 2016;34(27):3102-8.

15. Menniti-Ippolito F, Da Cas R, Traversa G, Santuccio C, Felicetti $P$, Tartaglia $L$, et al. Vaccine effectiveness against severe laboratoryconfirmed influenza in children: results of two consecutive seasons in Italy. Vaccine. 2014;32(35):4466-70.

16. Trotta F, Da Cas R, Spila Alegiani S, Gramegna M, Venegoni M, Zocchetti C, et al. Evaluation of safety of a/H1N1 pandemic vaccination during pregnancy: cohort study. BMJ. 2014;348:g3361.

17. Gliklich RE, Dreyer NA, Leavy MB. Registries for Evaluating Patient Outcomes: A User's Guide [Internet]. In: Adverse Event Detection, Processing, and Reporting. 3rd ed. Rockville (MD): Agency for Healthcare Research and Quality (US); 2014. Available from: https://www.ncbi.nlm.nih.gov/books/ NBK208615/.

18. Giambi C, Fabiani M, D’Ancona F, Ferrara L, Fiacchini D, Gallo T, et al. Parental vaccine hesitancy in Italy - Results from a national survey. Vaccine. 2018; pii: S0264-410X(17)31837-6. https://doi.org/10.1016/j. vaccine.2017.12.074.

19. Centers for Disease Control and Prevention, National Center for Immunization and Respiratory Diseases (NCIRD) Safety of Influenza Vaccines. https://www.cdc.gov/flu/professionals/vaccination/vaccine_safety.htm. Page last reviewed: August 24, 2017. Last accessed November 30, 2017.

20. World Health Organization (WHO). Causality assessment of adverse event following immunization (AEFI): user manual for the revised WHO. 2013.

21. Seo YB, Choi WS, Lee J, Song JY, Cheong HJ, Kim WJ. Comparison of the immunogenicity and safety of the conventional subunit, MF59-adjuvanted, 
and intradermal influenza vaccines in the elderly. Clin Vaccine Immunol. 2014;21(7):989-96.

22. Westphal DW, Williams SA, Leeb A, Effler PV. Continuous active surveillance of adverse events following immunisation using SMS technology. Vaccine. 2016;34:3350-5.

23. Leeb A, Regan AK, Peters IJ, Leeb C, Leeb G, Effler PV. Using automated text messages to monitor adverse events following immunisation in general practice. Med J Aust. 2014;200(7):416-8.

24. Regan AK, Blyth CC, Tracey L, Mak DB, Richmond PC, Effler PV. Comparison of text-messaging to voice telephone interviews for active surveillance of adverse events following immunisation. Vaccine. 2015;31(33):3689-94.

25. Crawford NW, Clothier H, Hodgson K, Selvaraj G, Easton ML, Buttery JP. Active surveillance for adverse events following immunization. Expert Rev Vaccines. 2014;13(2):265-76.

26. Lopalco PG, Johansen K, Ciancio B, De Carvalho GH, Kramarz P, et al. Monitoring and assessing vaccine safety: a European perspective. Expert Rev Vaccines. 2010;9(4):371-80.

27. Whitaker HJ, Ghebremichael-Weldeselassie Y, Douglas IJ, Smeeth L, Farrington CP. Investigating the assumptions of the self-controlled case series method. Stat Med. 2018;37(4):643-58.

28. Dodd CN, Romio SA, Black S, Vellozzi C, Andrews N, Sturkenboom M, et al. International collaboration to assess the risk of Guillain Barré syndrome following influenza a (H1N1) 2009 monovalent vaccines. Vaccine. 2013; 31(40):4448-58

29. Salmon DA, Proschan M, Forshee R, Gargiullo P, Bleser W, Burwen DR, et al. Association between Guillain-Barré syndrome and influenza a (H1N1) 2009 monovalent inactivated vaccines in the USA: a meta-analysis. Lancet. 2013; 381(9876):1461-8.

Ready to submit your research? Choose BMC and benefit from:

- fast, convenient online submission

- thorough peer review by experienced researchers in your field

- rapid publication on acceptance

- support for research data, including large and complex data types

- gold Open Access which fosters wider collaboration and increased citations

- maximum visibility for your research: over $100 \mathrm{M}$ website views per year

At $\mathrm{BMC}$, research is always in progress.

Learn more biomedcentral.com/submissions 